\title{
Inheritance of Susceptibility to Malignant Blood Disorders
} \author{
and Geir E Tjonnfjord ${ }^{1,2}$ \\ ${ }^{1}$ Department of Hematology, Oslo, Norway \\ ${ }^{2}$ Institute of Clinical Medicine, Oslo, Norway \\ ${ }^{3}$ Department of Research Computing, Oslo, Norway \\ ${ }^{4}$ Department of Computer Science, Copenhagen, Denmark \\ ${ }^{5}$ Norwegian Cancer Registry, Oslo, Norway \\ ${ }^{6}$ Department of Medicine, Torshavn, the Faroe Islands \\ ${ }^{7}$ Genetic Biobank, Torshavn, the Faroe Islands
}

Viggo Jonsson*1,2, Haneef Awan ${ }^{2,3}$, Neil D Jones ${ }^{4}$, Tom B Johannesen ${ }^{5}$, Bjarni a Steig ${ }^{6,7}$, Gudrid Andosdottir ${ }^{7}$

*Corresponding author: Viggo Jonsson, Department of Hematology, University of Oslo, Oslo University Hospital, Norway.

Received Date: March 18, 2019

Published Date: March 27, 2019

\section{Summary}

This is a study of familial malignant blood disease (MBD), both lymphoproliferative (LPD) and myeloproliferative (MPD). One group is from Norway and Denmark: 112 families with unrelated parents, in all 301 cases of MBD. This group is compared with 315 patients with MBD from the Faroe Islands. The Faroese population is in effect one large inbred family with related parents. LPD includes the lymphoid leukemias (chronic and acute), the malignant lymphomas (morbus Hodgkin and non-Hodgkin's lymphomas), multiple myeloma and a few other diagnoses. MPD includes the myeloid leukemias (chronic and acute), myelodysplasia, myelofibrosis, polycythemia vera and essential thrombocytosis.

For each patient (proband, P) we have counted in the pedigree the relatives with MBD (affected relatives, AR), including diagnosis, age, gender, year of onset of disease, and the position of each AR in paternal or maternal line. We applied pattern recognition (a biostatistical technique to recognize patterns in large data), in particular "graphical models". This revealed a very clear picture of the mutual distribution of MBD along 3 or 4 generations of the family trees.

In families with unrelated parents, the genealogical combination of affected parent-affected child is most frequent, in particular the combination affected mother-affected son. This holds for both LPD and MPD, and in their combinations. In contrast, there were almost no affected parent-affected offspring combinations in the large consanguineous family; but on the other hand, so-called oblique combinations of affected child-affected aunt or -affected uncle were seen. If we regard the Faroese population as one large family, every proband has sufficiently many AR so that their distribution along paternal and maternal lines can be recorded. In the Faroese material, chronic lymphocytic leukemia (CLL) and multiple myeloma are equally frequent and comprise the most MBD. Acute myeloblastic leukemia is the most frequent type of MBD. The ratio LPD/MPD is 2.5/1, and the ratio of men to women is 1.5/1 for both LPD and MPD.

A systematic study of the relationship between every proband and its AR reveals three significant patterns (pathways) in the transgenerational segregation of susceptibility (risk genes): (I) An increased number of male AR in paternal lines to male $\mathrm{P}$, and a decreased number of male AR to female $P$ in paternal lines $(\mathrm{P}<0.05$ to 0.001$)$. The number of observed female $A R$ is not significantly different from the expected number of female AR along either paternal or maternal lines $(\mathrm{P}>0.05)$. Multiple myeloma is prototypical. (II) An increased number of male AR to male P and a decreased number of male AR to female P in both paternal and maternal lines $(\mathrm{P}<0.05$ to 0.001$)$. Chronic lymphocytic leukemia is prototypical. (III) The number of male AR and female AR is not significantly different from the expected number in paternal and maternal lines $(\mathrm{P}>0.05)$. Acute lymphoblastic leukemia and nonHodgkin's lymphomas are prototypical. 
Further, in the Faroese material, we have observed anticipation (increasing aggressiveness of MBD down through the generations, i.e., increased occurrence of malignancy and progressively lower average age at debut of disease). For instance, the frequencies of acute lymphoblastic leukemia and of multiple myeloma are significantly higher on the Faroe Islands than in the Norwegian and Danish populations, as seen by comparing data from the Faroe Islands with data from the Norwegian and Danish cancer registries. Male patients consistently have a significantly lower average age at onset of MBD than female patients $(\mathrm{P}<0.05)$.

Some questions are inevitable: what are the inheritance patterns of MBD? How can these numerous and diverse data be integrated to yield a meaningful explanation of how transgenerational segregation of susceptibility genes occurs? What explains the distribution of LPD and MPD in the family tree? One must of course take account of the limitations of the material investigated. Even if all patients and all patient files were thoroughly checked and compared with information from the cancer registries, it is unavoidable that some patients with low-grade disease or no or only few symptoms can be overlooked. Thus, one must assume in advance that the investigated material is under-registered. On the other hand, we may assume that the genealogical data are very reliable, and that he family trees used are correct on the whole. Each proband's relatives have been double-checked with the National Person Registries in Norway, Denmark or the Faroe Islands; and this also applies to their healthy relatives.

It was immediately apparent that traditional Mendelian segregation was not an explanation. We also included previously published articles on MBD, including papers about birth-order effect (the rank number by age of the proband in the sib ship), and found by means of pattern recognition that both maternal parental imprinting (segregating paternal transgenerational lines of pairs of affected father-affected son) and paternal parental imprinting (segregating maternal transgenerational lines of pairs of affected mother-affected daughter) were operating. We discuss whether, from a genetic viewpoint, this is the background for the pronounced clinical difference between male and female chronic lymphocytic leukemia, e.g. a worse prognosis in male than in female CLL; and the surplus of male CLL. The patterns of segregation we saw imply that both maternal and paternal imprinting are operating in a concurrent balance providing both pairs of affected father-affected son or affected daughter (maternal imprinting) and pairs of affected mother-affected daughter or affected son. All known systems of socalled meiotic drive (non-Mendelian segregation) result in a male/ female ratio different from $1 / 1$, and in fact one sees an excess of males in most cases of MBD, e.g. a 1.5/1 ratio in CLL.

Such parentally imprinted genes are known to be master genes of growth factors, in casu growth factors related to monoclonal growth of the early fetal hematopoietic stem cells.; and such parentally imprinted genes are known to be regulated by modifier genes. Pattern recognition suggests a regulator in the form of mother-son microchimerism, presumably one that depends on HLA-matches between the mother, her children, the father, the number of the mother's earlier male partners, and mother's parity. Accordingly, microchimerism is well-described as a mechanism underlying the development of autoimmunity, graft-versusleukemia effect, and birth-order effect, all of which occur in MBD.

Microchimerism leads to an increase of mother-offspring derived genomic diversity. In each generation, a larger and larger load of genomic diversity is accumulated in MBD. Anticipation describes this gradual accumulation of genetic susceptibility and increasing malignancy. These observed patterns also reveal a mechanism that, in spite of the gradual increase, prevents overload of susceptibility in the family: More and more malignancy develops down through the generations, eventually leading to a situation where probands die of high-malignant disorders in childhood or in youth before fertile age, which thereby depletes the susceptibility of the family and prevents overload.

\section{Acknowledgement}

None.

\section{Conflict of Interest}

No conflict of interest.

\section{References}

1. Jonsson V, Awan H, Jones ND, Johannesen TB, a Steig B, Andosdottir G, Tjonnfjord GE. Nature.Com Scientific Reports (2019). 